\title{
Condición física en jóvenes y su relación con la actividad física escolar y extraescolar
}

\author{
Fátima CHACÓN-BORREGO \\ Juan Antonio CORRAL-PERNÍA \\ Carolina CASTAÑEDA VÁZQUEZ
}

Datos de contacto:

Fátima Chacón Borrego Universidad de Sevilla

fchacon@us.es

Juan Antonio Corral Pernía

Universidad de Sevilla

juancorral@us.es

Carolina Castañeda Vázquez

Universidad de Sevilla

carolinacv@us.es

\section{RESUMEN}

Los niveles de actividad física y condición física se relacionan con diferentes parámetros de salud en adolescentes. Asimismo, la condición física es un contenido del currículo educativo de Educación Física. Sin embargo, la evidencia científica muestra que los niveles de actividad física de los escolares son insuficientes para obtener mejoras en la salud. La investigación pretende conocer el nivel de condición física según el sexo y la edad de los adolescentes, y analizar las posibles relaciones entre la condición física, la actividad-inactividad de los adolescentes y la práctica deportiva competitiva extraescolar.

Se evaluó una muestra de 196 adolescentes: 104 mujeres (47\%) de 13-17 años $(14 \pm 1,17)$ de Sevilla (España) mediante el Cuestionario de actividad física para niños (PAQ-A) y la batería ALPHA-Fitness. Se registró el índice de masa corporal, perímetro de cintura y la capacidad músculo-esquelética y aeróbica, (dinamometría manual, salto de longitud y test de Course Navette). Los resultados muestran que los hombres presentan valores más altos en la prueba de Course Navette, dinamometría manual y salto de longitud. La práctica deportiva extraescolar se asocia con niveles más altos en salto de longitud $(p<.05)$ y Course Navette $(p<.01)$. Se mantienen las diferencias de género, los niños presentaron valores más altos en todas las pruebas. Los estudiantes que practican deporte competitivo extraescolar presentan mejor condición física, especialmente muscular y cardiorrespiratoria. Deben promoverse programas de práctica deportiva extraescolar en las escuelas, ya que puede ser una medida efectiva para mejorar la condición física y la salud en general.

PALABRAS CLAVE: Adolescentes; Condición física; Salud; Deporte; Alpha Fitness; Educación Secundaria. 


\title{
Physical fitness in youngsters and its relationship with school and extracurricular physical activity
}

\begin{abstract}
High levels of physical activity and physical fitness are related to different health parameters in adolescents. Likewise, physical fitness is a content within the educational curriculum of Physical Education in Primary Education and, especially, in Secondary Education. Nevertheless, evidence shows that physical activity levels of schoolchildren and especially from Secondary Education, are insufficient to obtain improvements in health. The main aim of the research is to report sex and age specific physical fitness levels in adolescents, and analyze the possible relationships between physical fitness, active-inactive teenagers and extracurricular sport practice. A sample of 196 adolescents (104 women, $47 \%$ ), aged $13-17$ years $(14 \pm 1,17)$ from Seville (Spain) was assessed by Physical Activity Questionnaire for Adolescents (PAQ-A) and the ALPHA-Fitness Battery. The autors assessed body mass index, waist circumference, and also muscular fitness and cardiorrespiratory fitness, using handgrip, standing long jump and 20-m shuttle run test. Results show that males have on average higher values in the 20-m shuttle run test, manual dynamometry and longitudinal jump. The analysis showed that extracurricular sport practice is directly associated with higher levels in long jump $(p<.05)$ and 20 -m shuttle run test ( $p$ $<.01$ ). Gender differences in the physical fitness are maintained, boys presented higher values in all selected tests. Students who practice extracurricular sport are more likely to have high levels of physical fitness, especially muscular and cardiorrespiratory fitness. Programs promoting extracurricular sport practice should be offered and developed at schools, since it can be effective measure to improve physical fitness and therefore overall health.
\end{abstract}

KEYWORDS: Adolescents; Physical fitness; Health; Sport; Alpha Fitness; Secondary Education.

\section{Introducción}

La condición física $(\mathrm{CF})$ es un contenido dentro del currículo educativo de Educación Física en Educación Primaria y fundamentalmente en Educación Secundaria, que está relacionado con la mejora de la salud y la calidad de vida de los escolares. Su inclusión en estas etapas educativas es de especial importancia teniendo en cuenta que uno de sus objetivos es desarrollar en los escolares estilos de vida saludables basados en la actividad fisica (AF) que puedan permanecer durante la edad adulta (Cuenca-García et al., 2011; Martínez-Vizcaíno \& Sánchez-López, 2008).

Esto lleva al planteamiento de las siguientes cuestiones: los adolescentes ¿tienen una buena $\mathrm{CF}$ ? ¿Existen diferencias de $\mathrm{CF}$ en función de la AF realizada en el ámbito escolar o extraescolar?

La CF define el estado o capacidad que tiene una persona para realizar 
todo tipo de actividades fisicas, las vinculadas con tareas habituales y/o deportivas (Merino \& González, 2010; Ortega, Gutiérrez, Meusel, Sjöström, $\&$ Castillo, 2006).

En este sentido, tener una buena $\mathrm{CF}$ desde un parámetro de salud, implica tener una buena capacidad cardiorespiratoria, de fuerza y resistencia muscular, de flexibilidad, y una adecuada composición corporal, sin un exceso de grasa (Martínez-Vizcaíno \& Sánchez-López, 2008; Ortega et al., 2013), considerándose como un factor protector de numerosas enfermedades asociadas, en gran medida, a la inactividad y el sedentarismo.

Diferentes investigaciones sobre niños y adolescentes han constatado que altos niveles de CF significa, desde un punto de vista físico, una mejor salud cardiovascular, metabólica y musculo-esquelética. Ésta, a su vez, se relaciona con una mejor capacidad aeróbica, menores niveles de sobrepeso y obesidad, mejores valores de mineralización de los huesos y una mayor masa muscular (Muntaner-Mas, Vidal-Conti, Cantallops, Borràs, \& Palou, 2017; Kim et al., 2005; Merino \& González, 2010; Ortega et al., 2013; Rush et al., 2014; Secchi, Garcia, España-Romero \& Castro-Piñero, 2014).

Al mismo tiempo, se ha comprobado que estas mejoras de la $\mathrm{CF}$ en escolares también sirven como mecanismo protector de enfermedades en la edad adulta, siendo considerado como un factor determinante en la longevidad y calidad de vida de las personas (Balady, 2002; Boreham et al., 2002; Laukkanen et al., 2001; Seguin \& Nelson, 2003).

El desarrollo y mejora de estas capacidades está vinculado fundamentalmente a la realización de ejercicio físico (Ortega, Ruiz, HurtigWennlöf \& Sjöström, 2008; Ruiz et al., 2009) y por tanto, al cumplimiento de las recomendaciones existentes de AF para niños y adolescentes en cuanto a frecuencia, intensidad y tipo de ejercicio, de al menos 60 minutos de AF moderada y vigorosa de resistencia aeróbica, de tres dias de actividades de fortalecimiento muscular y mejora de masa ósea y reducción de comportamientos sedentarios a un máximo de dos horas al día (Organización Mundial de la Salud, 2010).

Según se exponía anteriormente, el principal medio de mejora de la CF es la realización de AF para lo cual, en niños y adolescentes, el contexto escolar, la clase de educación física, los recreos e incluso otras asignaturas, pueden jugar un papel esencial en incrementar la actividad y conseguir umbrales de intensidad y duración suficiente para la mejora de estas capacidades (Carredo \& Cecchini, 2019; Ardoy et al., 2011; Cuenca-García et al., 2011; Merino \& González, 2010). Igualmente es necesario realizar actividades fuera del centro educativo, tanto actividades deportivas extraescolares, como otras actividades físicas de la vida cotidiana en las que se incluyan los desplazamientos activos (Fundación para la investigación nutricional, 2018).

Sin embargo, existen numerosas evidencias de que la $\mathrm{AF}$ de los escolares y sobre todo a partir de la Educación Secundaria, es insuficiente 
para obtener mejoras desde un punto de vista de salud (Sallis, 2000).

Según datos de la Fundación para la investigación nutricional (2018) y estudio ANIBES (Ruiz et al., 2015), los adolescentes de 13 a 16 años y de 17-18 años que cumplen con las recomendaciones de AF son el 50\% y el $22 \%$ respectivamente; en las adolescentes estas cifras son mucho más alarmantes, el 14\% (en el tramo de edad 13-16) y el 8\% (17-18 años). En este mismo sentido, Guthold, Stevens, Riley \& Bull (2019), indican que el $76,6 \%$ de los escolares españoles de 11 a 17 años realizan una insuficiente AF (datos relativos a 2016).

Así mismo, la investigación de Ortega et al. (2005) sobre los niveles de CF de los adolescentes muestra como la CF en España es inferior a la de otros países. Incluso en la actualidad se ha observado en esta etapa de la adolescencia, un empeoramiento con respecto a décadas anteriores en algunas capacidades físicas como la aeróbica de acuerdo con los resultados obtenidos por Tomkinson, Léger, Olds \& Cazorla (2003); para Eisenmann y Malina (2002) este empeoramiento se produce sobre todo a partir de los 15 años. En función del género, las niñas y adolescentes, además de ser menos activas, tienen peores resultados que el género masculino en los test de $\mathrm{CF}$ de resistencia cardiovascular, fuerza del tren superior e inferior y velocidad, a excepción de en las pruebas de flexibilidad (Arriscado, Muros, Zabala, \& Dalmau, 2014; Secchi et al., 2014).

De acuerdo con las investigaciones, una escasa AF y una baja CF puede tener repercusiones importantes en la salud de los adolescentes.

Teniendo en cuenta lo expuesto, los objetivos del presente estudio son: conocer las diferencias de CF de estudiantes de Secundaria en función del género y la edad. Analizar la relación de la $\mathrm{CF}$ con el nivel de actividadinactividad y con la realización de deporte extraescolar competitivo.

\section{Método}

Se trata de una investigación con un diseño no experimental de corte transversal y descriptivo.

La muestra, seleccionada por muestreo incidental, por la facilidad de contacto y acceso con determinados centros educativos, está formada por 196 sujetos: 104 mujeres (47\%) y 92 hombres (53\%), de entre 13 y 17 años $(14 \pm 1,17)$.

Para analizar el nivel de AF de los adolescentes se utilizó el PAQ-A (Physical Activity Questionnaire for Adolescents), traducido y validado al español por Martínez-Gómez et al. (2009). Cuestionario de 9 items medidos con una escala tipo Likert 1-5, en el que contabilizan las puntuaciones obtenidas en las 8 primeras preguntas: " 2 . En los últimos 7 días, durante las clases de educación física, ¿cuántas veces estuviste muy activo durante las clases: jugando intensamente, corriendo, saltando, haciendo lanzamientos? Opciones de respuesta: Valor 1: No hice/hago educación física; 2: Casi nunca; 3: Algunas veces; 4: A menudo; 5: Siempre. A1 cuestionario se le añadió una pregunta para saber si el alumnado realizaba 
práctica deportiva extraescolar de carácter competitivo.

Para medir la CF se emplearon diversas pruebas de la Batería ALPHAFitness (Ruiz et al., 2011.), en su versión de alta prioridad. Para la realización de las pruebas se siguió la secuencia recomendada por el manual de instrucciones de la batería, desarrollándose a lo largo de 2-3 sesiones en el orden que se explicita a continuación: Peso y altura (IMC); Perímetro de cintura; Fuerza de prensión manual; Salto en longitud a pies juntos y Test de ida y vuelta de $20 \mathrm{~m}$.

De manera previa a toda la toma de datos se mantuvo una reunión con los equipos directivos de los centros para informales del proyecto y se les solicitó autorización para desarrollarlo. Posteriormente, se informó a los Claustros de Profesores, a las familias y al alumnado de las pruebas y del periodo en el que se iban a realizar. Se entregó una carta informativa junto al consentimiento informado para las familias, debiendo entregar el alumnado que quisiera participar en la investigación el correspondiente consentimiento informado, firmado por su padre, madre o tutor legal, al ser estudiantes menores de edad. La participación en cualquier caso fue voluntaria y se les aseguró el anonimato de los datos que aportaban a través del establecimiento de códigos en los cuestionarios.

Finalmente se procedió a la toma de datos a través de los cuestionarios y a la valoración de las diferentes pruebas desarrolladas de la Batería ALPHA-Fitness, con el siguiente instrumental:

El peso se registró a través de una báscula de bioimpedancia electrónica OMRON BF511. Para la talla se empleó un tallímetro y para el perímetro de cintura una cinta ergonómica flexible, no elástica. La fuerza de prensión manual se midió con un test de dinamometría manual empleando un dinamómetro digital modelo TKK5401, con adaptador de amplitud del agarre incorporado (España-Romero et al., 2010). Para la medida del salto de longitud a pies juntos se utilizó una cinta métrica de PVC y fibra de vidrio (Modelo 74-Y100M, CST/Berger, Chicago, USA). En el test de Course-Navette se empleó un equipo audio portátil y un dispositivo de memoria USB.

En el análisis de los datos se aplicó estadística cuantitativa de carácter descriptivo. A través del programa SPSS versión 26.0 se realizó un análisis descriptivo de las variables a través de frecuencias y porcentajes, medias y desviaciones típicas. Se realizaron pruebas de normalidad, determinando que los datos no seguian una distribución normal, por lo que se emplearon contrastes de hipótesis no paramétricos (Prueba U de Mann-Whitney y Rho de Spearman) para evaluar la diferencia significativa entre las medias de los grupos según género y la posible correlación entre variables. El nivel de significación se fijó a $\mathrm{p} \leq 0.05$.

\section{Resultados}

La tabla 1 muestra los resultados descriptivos de los participantes en la investigación, diferenciados en sus respectivas categorias según el sexo, en 
las pruebas de la bateria ALPHA-Fitness de alta prioridad. Los puntajes se encuentran categorizados en los 5 grupos establecidos en el manual de la batería: "muy bajo", "bajo", "medio", "alto" y "muy alto", establecidos a su vez previamente según los baremos de edad y sexo.

Tabla 1

Puntuaciones en las pruebas de ALPHA-Fitness según sexo

\begin{tabular}{|c|c|c|c|c|c|c|c|c|}
\hline \multirow{2}{*}{$\begin{array}{c}\text { Pruebas } \\
\text { ALPHA-Fitness }\end{array}$} & \multirow{2}{*}{ Sexo } & $\begin{array}{c}\text { Chi- } \\
\text { cuadrado }\end{array}$ & \multicolumn{5}{|c|}{ Categorias ALPHA Fitness } & \multirow[b]{2}{*}{ Total } \\
\hline & & (a) & $\begin{array}{c}\text { Muy } \\
\text { bajo \% }\end{array}$ & $\begin{array}{c}\text { Bajo } \\
\%\end{array}$ & $\begin{array}{c}\text { Medio } \\
\%\end{array}$ & $\begin{array}{c}\begin{array}{c}\text { Alto } \\
\%\end{array} \\
\end{array}$ & $\begin{array}{c}\text { Muy } \\
\text { alto \% }\end{array}$ & \\
\hline \multirow[t]{2}{*}{ IMC } & Mujer & 0,09 & 8,7 & 14,6 & 44,7 & 16,5 & 15,5 & 100 \\
\hline & Hombre & & 19,8 & 12,1 & 49,5 & 9,9 & 8,8 & 100 \\
\hline \multirow{2}{*}{$\begin{array}{l}\text { Perimetro } \\
\text { cintura }\end{array}$} & Mujer & 0.00 & 37,5 & 14,6 & 29,2 & 13,5 & 5,2 & 100 \\
\hline & Hombre & & 9,3 & 27,9 & 51,2 & 8,1 & 3,5 & 100 \\
\hline \multirow[t]{2}{*}{ Dinamometria } & Mujer & 0,00 & 54,5 & 23,2 & 8,1 & 7,1 & 7,1 & 100 \\
\hline & Hombre & & 25,3 & 27,6 & 24,1 & 16,1 & 6,9 & 100 \\
\hline \multirow[t]{2}{*}{ Salto longitud } & Mujer & 0,00 & 17,7 & 17,7 & 18,8 & 32,3 & 13,5 & 100 \\
\hline & Hombre & & 8,3 & 35,7 & 28,6 & 14,3 & 13,1 & 100 \\
\hline \multirow[t]{2}{*}{ Course Navette } & Mujer & 0,01 & 21,9 & 15,6 & 21,9 & 12,5 & 28,1 & 100 \\
\hline & Hombre & & 17,9 & 6,0 & 9,0 & 28,4 & 38,8 & 100 \\
\hline
\end{tabular}

Se observa en la tabla anterior (Tabla 1) que las mujeres presentan valores altos y muy altos de IMC (32\%) en mayor proporción que los hombres $(18,7 \%)$, aunque estas diferencias no alcanzan a ser significativas.

En cuanto a los valores medios del perímetro de cintura, el grupo de las chicas presenta un porcentaje más elevado en valores altos y muy altos, los vinculados con el riesgo de sobrepeso y obesidad, $(18,7 \%)$ que los chicos $(11,6)$. No obstante, también son ellas quienes presentan igualmente valores bajos y muy bajos $(52,1 \%)$ de perímetro de cintura, mientras que la mitad de los hombres $(51,2 \%)$ presenta valores normales. En estos casos, la diferencia en la distribución de los niveles según sexo sí resulta significativa, encontrándose ellas en mayor medida por debajo y por encima de la categoría central, y ellos en mayor medida en la categoría "media".

Los hombres presentan porcentajes más altos en la dinamometría manual que las mujeres, siendo las diferencias entre grupos también significativas. Así, en las categorías establecidas, el $77 \%$ de las mujeres presenta valores bajos y muy bajos, mientras que el $23 \%$ de los hombres presenta valores altos y el $24,1 \%$ medios.

En el salto de longitud no obstante, a nivel general, las mujeres 
presentan una capacidad músculo-esquelética de miembros inferiores algo más alta que los hombres, pues los adolescentes que están en las categorias medias y altas son el $56 \%$ en el sexo hombres frente al $64,6 \%$ en las mujeres.

En el test de Course Navette, sin embargo, los hombres presentan una mayor capacidad aeróbica, ya que las mujeres se encuadran en los valores más bajos $(37,5 \%)$ o medios $(21,9 \%)$, mientras que los hombres se encuadran en mayoría $(67,2 \%)$ en los valores más altos, que junto con los valores medios representa el $76,2 \%$ de la muestra.

Tabla 2

Resultados descriptivos de pruebas de condición física por grupos según edad $y$ sexo

\begin{tabular}{|c|c|c|c|c|c|c|c|c|c|c|c|c|c|c|c|c|}
\hline \multicolumn{2}{|l|}{$y$} & \multicolumn{3}{|c|}{ IMC } & \multicolumn{3}{|c|}{ PER. CINTURA } & \multicolumn{3}{|c|}{ DINAMOMETRÍA } & \multicolumn{3}{|c|}{ SALTO LONG. } & \multicolumn{3}{|c|}{ C. NAVETTE } \\
\hline Edad & Sexo & M & DT & SIG. & M & DT & SIG. & M & DT & SIG. & M & DT & SIG. & $\mathbf{M}$ & DT & SIG. \\
\hline \multirow[b]{2}{*}{13} & $\mathrm{H}$ & 20,5 & 4,7 & \multirow{2}{*}{0,36} & 70,6 & 10,2 & \multirow{2}{*}{0,81} & 22,6 & 6,7 & \multirow[b]{2}{*}{0,76} & 163,5 & 19,7 & \multirow[b]{2}{*}{$0,01^{*}$} & 5,9 & 2,3 & \multirow[b]{2}{*}{$0,00^{*}$} \\
\hline & M & 22,2 & 4,9 & & 70,0 & 10,2 & & 21,6 & 4,2 & & 142,4 & 28,2 & & 3,3 & 1,6 & \\
\hline \multirow{2}{*}{14} & $\mathrm{H}$ & 20,7 & 3,3 & \multirow{2}{*}{$0,04^{*}$} & 71,4 & 5,9 & \multirow{2}{*}{0,39} & 29,6 & 4,4 & \multirow[b]{2}{*}{$0,00^{*}$} & 175,0 & 18,3 & \multirow{2}{*}{$0,00^{*}$} & 6,4 & 3,5 & \multirow{2}{*}{0,14} \\
\hline & M & 23,2 & 3,7 & & 70,4 & 7,7 & & 20,9 & 4,2 & & 143,3 & 32,4 & & 2,8 & 1,3 & \\
\hline \multirow{2}{*}{15} & $\mathrm{H}$ & 21,5 & 3,7 & \multirow{2}{*}{0,27} & 74,5 & 6,8 & \multirow{2}{*}{$0,00^{*}$} & 35,7 & 6,3 & \multirow{2}{*}{$0,00^{*}$} & 189,6 & 19,2 & \multirow{2}{*}{$0,00^{*}$} & 7,4 & 2,6 & \multirow{2}{*}{$0,00^{*}$} \\
\hline & M & 22,6 & 4,7 & & 68,3 & 9,3 & & 23,6 & 5,5 & & 150,1 & 22,5 & & 4,5 & 2,2 & \\
\hline \multirow[b]{2}{*}{16} & $\mathrm{H}$ & 22,7 & 3,1 & \multirow[b]{2}{*}{0,35} & 74,7 & 4,8 & \multirow[b]{2}{*}{$0,01^{*}$} & 38,6 & 5,2 & \multirow[b]{2}{*}{$0,00^{*}$} & 195,9 & 17,7 & \multirow[b]{2}{*}{$0,00^{*}$} & 8,6 & 2,1 & \multirow{2}{*}{$0,00^{*}$} \\
\hline & M & 22,1 & 3,2 & & 68,2 & 7,7 & & 21,8 & 6,7 & & 150,18 & 19,1 & & 4,8 & 2,4 & \\
\hline \multirow[b]{2}{*}{17} & $\mathrm{H}$ & 24,4 & 2,9 & \multirow[b]{2}{*}{$0,04^{*}$} & 74,6 & 6,0 & \multirow[b]{2}{*}{0,07} & 43,3 & 9,2 & \multirow[b]{2}{*}{$0,00^{*}$} & 200,0 & 38,9 & \multirow[b]{2}{*}{$0,01^{*}$} & 7,2 & 2,6 & \\
\hline & M & 21,8 & 0,6 & & 67,5 & 4,2 & & 21,2 & 3,1 & & 142,8 & 18,8 & & 4,6 & 2,3 & $0,05^{*}$ \\
\hline
\end{tabular}

H: Hombre; M: Mujer; M: Media; DT: Desviación típica; SIG.: Sig. Asintótica (bilateral) por U de Mann-Whitney.

El análisis por grupos de edad y sexo mostrado en la tabla anterior (Tabla 2) aporta datos más concretos de la población analizada y las diferencias entre sexos. Así, en cuanto a los datos de IMC y perímetro de cintura, se observan diferencias de medias entre sexo estadisticamente significativas ( $\mathrm{p} \leq 0.05)$ en casos concretos. Por ejemplo, en el IMC, en el grupo de edad de 14 años, las mujeres presentan una media más alta que los hombres (23,2 y 20,7 respectivamente), mientras que en el grupo de edad de los 17 años, son los hombres quienes presentan una media de IMC más alta que las mujeres $(24,4$ frente a 21,8$)$. En el resto de los casos las diferencias entre grupos no son significativas. En el caso del perimetro de cintura, tanto en los grupos de 15 y 16 años, son los chicos quienes presentan una media de perímetro superior a las chicas (en 15 años 74,5 frente a 63,3 y en 16 años 74,8 frente a 68,2), siendo solo en estas edades significativa la diferencia de medias.

Respecto al resto de pruebas: dinamometría, salto de longitud y Course Navette, la diferencia de medias de cada grupo por edad y sexo muestra, de manera general, que los chicos presentan mejores valores de CF que las chicas. De este modo, en la dinamometría, desde los 14 años incluidos, los hombres presentan valores medios superiores a las mujeres, siendo las diferencias significativas ( $\mathrm{p} \leq 0.05$ ), y demostrando así mayor capacidad músculo-esquelética en miembro superior (en 14 años 29,6 frente a 20,9; en 15 años 35,7 frente a 23,6; en 16 años 38,6 frente a 21,8 y en 17 años $43,3$ frente a 21,2$)$. 
En el salto de longitud, en todos los grupos de edades, los chicos presentan medias superiores a las chicas de manera significativa $(p \leq 0.05)$, señalando así mayor capacidad músculo-esquelética en el tren inferior (en 13 años 163,5 frente a 142,4; en 14 años 175,0 frente a 143,3; en 15 años 189,6 frente a 150,1 ; en 16 años 195,9 frente a 150,18 y en 17 años 200,0 frente a 142,8$)$.

En el Course Navette los resultados son muy similares, todos los grupos de edad, excepto los 14 años, muestran diferencias de medias significativa ( $\mathrm{p} \leq 0.05$ ) según el sexo, en la que los hombres muestran una mayor capacidad aeróbica que las mujeres (en 13 años 5,9 frente a 3,3; en 15 años 7,4 frente a 4,5; en 16 años 8,6 frente a 4,8 y en 17 años, 7,2 frente a 4,6).

Los datos recogidos señalaron también que el $10 \%$ de los adolescentes encuestados realizaba suficiente AF como para ser clasificado según el PAQ-A como activos, mientras que el $90 \%$ se clasifica como inactivo.

En la tabla 3 se señalan las correlaciones (a través de Rho de Spearman) entre las puntuaciones obtenidas en las diferentes pruebas de ALPHAfitness y la clasificación del alumnado en activo-inactivo según el PAQ-A.

Tabla 3

Correlaciones entre actividad e inactividad fisica y las puntuaciones en las pruebas de ALPHA-Fitness

\begin{tabular}{|c|c|c|c|c|c|c|}
\hline & & IMC & $\begin{array}{c}\text { Perimetro } \\
\text { Cintura }\end{array}$ & Dinamometria & $\begin{array}{c}\text { Salto } \\
\text { longitud }\end{array}$ & $\begin{array}{c}\text { Course } \\
\text { Navette }\end{array}$ \\
\hline \multirow{3}{*}{$\begin{array}{l}\text { Clasificación } \\
\text { PAQ-A } \\
\text { ACTIVO- } \\
\text { INACTIVO }\end{array}$} & Coef. correlación &,- 068 &,- 119 &,- 049 & ,038 &, 140 \\
\hline & Sig. (bilateral) &, 343 &, 109 &, 503 &, 612 &, 111 \\
\hline & $\mathrm{N}$ & 194 & 182 & 186 & 180 & 131 \\
\hline
\end{tabular}

Los datos de la tabla anterior (Tabla 3) reflejan que no existe correlación entre la actividad-inactividad fisica del alumnado $y$ las diferentes puntuaciones obtenidas por los adolescentes en las pruebas de la batería ALPHA-Fitness.

Respecto al alumnado que realizaba práctica deportiva competitiva fuera del horario lectivo, resultó que el 29,3\% de los encuestados participaba en algún tipo de AF competitiva en el horario extraescolar.

En la tabla 4 se señalan las correlaciones (a través de Rho de Spearman) entre las puntuaciones obtenidas en las diferentes pruebas de ALPHAfitness y la práctica deportiva extraescolar competitiva. 
Tabla 4

Correlaciones entre la práctica deportiva competitiva extraescolar y las puntuaciones en las pruebas de ALPHA-Fitness

\begin{tabular}{llccccc}
\hline & & IMC & $\begin{array}{c}\text { Perimetro } \\
\text { Cintura }\end{array}$ & Dinamometria & $\begin{array}{c}\text { Salto } \\
\text { longitud }\end{array}$ & $\begin{array}{c}\text { Course } \\
\text { Navette }\end{array}$ \\
\hline $\begin{array}{l}\text { Compite } \\
\text { en algún } \\
\text { deporte }\end{array}$ & Coef. correlación &,- 076 &,- 099 &, 121 &, $175^{*}$ &, $314^{* *}$ \\
\cline { 2 - 7 } & Sig. (bilateral) &, 293 &, 184 &, 100 &, 019 &, 000 \\
\cline { 2 - 7 } & $\mathrm{N}$ & 193 & 181 & 185 & 179 & 130 \\
\hline
\end{tabular}

Rho de Spearman. **La correlación es significativa en el nivel 0,01 (bilateral); *La correlación es significativa en el nivel 0,05 (bilateral).

Los datos dejan entrever que existe una correlación positiva significativa entre la práctica deportiva extraescolar competitiva y el salto de longitud $\left(0,175^{*}\right)$, es decir, la capacidad músculo-esquelética del tren inferior; e igualmente una correlación positiva en este caso muy significativa, entre la práctica deportiva extraescolar competitiva y el test de Course Navette $\left(0,314^{* *}\right)$, es decir, la capacidad aeróbica. Sin embargo, no tiene ninguna relación con el perímetro de cintura y el IMC, y por tanto con la grasa corporal.

\section{Discusión}

Los resultados obtenidos en el presente estudio instan a considerar, en primer lugar, la importancia que requiere el segmentar los resultados por edad y sexo, dado que en las edades analizadas son muchas las variables que pueden incidir en un aumento o disminución de los valores presentados, desde las fases sensibles de desarrollo de las cualidades físicas, hasta los hábitos y estilos de vida predominantes que puedan incidir en la mismas, aunque aun asî nos hayamos centrado principalmente en la última fase de la adolescencia temprana y la denominada adolescencia media, pues son muchos los autores que muestran sus resultados como medias de una etapa, sin hacer distinción por edad, tal y como recogen Ortega et al. (2005) en los valores normativos de los adolescentes españoles.

Dicha diversidad metodológica, requiere la realización de una interpretación orientativa de las comparaciones realizadas.

De esta manera, se puede indicar que el análisis a nivel condicional realizado nos muestra diferencias significativas en casi todas las edades en ambos géneros, excepto en las medidas de la composición corporal, la cual señala una relación directamente proporcional en relación a la edad, coincidiendo con lo indicado por Bucco-dos Santos \& Zubiaur-González (2013), siendo mayor en las mujeres que en los hombres hasta los 15 años, edad a partir de la cual se invierten los valores, siendo mayores los valores de los hombres.

Así pues, se muestra la concordancia de los resultados de la presente investigación con estudios como los de Ortega et al. (2005) y Catley \& 
Tomkinson (2013), en relación a los resultados superiores obtenidos en el género masculino a nivel condicional, pudiendo atribuirse a un mayor desarrollo de la masa muscular, ya que se entiende que la coordinación neuromuscular debe ser similar e incluso superior en el género femenino en las primeras edades dado su desarrollo madurativo.

Por otro lado, son numerosos los estudios que muestran la relación inversa existente entre la $\mathrm{CF}$, específicamente la capacidad aeróbica y la fuerza muscular, con la grasa corporal total (Ortega et al. 2007, Ortega et al., 2010), aunque dado que la presente evaluación se basa en el IMC, no resulta conveniente realizar comparación alguna, dada la diferencia de variables y, más aun teniendo en cuenta lo indicado relativo al desarrollo evolutivo en estas edades, donde un aumento de la masa muscular junto con una disminución de masa grasa, pudieran no reflejar variación alguna en el IMC, cuando sí existe cambio en la composición corporal. No obstante, los resultados de IMC presentados van en consonancia a los obtenidos en estudios nacionales como los de Nieto, García, \& Guillamón (2020); Cuenca-García et al. (2011), Moreno et al. (2005) y Serra-Majem et al. (2003).

En relación a la $\mathrm{CF}$, esta investigación ha mostrado resultados superiores a los registrados por Martínez, de los Reyes-Corcuera, BorrellLizana \& Pastor-Vicedo (2018), siendo algo normal atendiendo al desarrollo de la CF según la edad, dado que se centran en alumnado de 11-12 años. Respecto al IMC, se observa similitud en el género masculino, mientras que esta investigación ha registrado un IMC superior en las mujeres, y un perímetro de cintura inferior en ambos sexos, pudiendo deberse a un aumento de la masa muscular en estas edades.

Sin embargo, si se comparan los resultados en salto de longitud con el estudio de Secchi et al. (2014), los datos del presente estudio son superiores en el género femenino en todas las edades analizadas, también lo son en el tramo de edad de 13 años en los hombres, tampoco existe coincidencia con el estudio de Sylejmani et al. (2019).

Los resultados obtenidos en el test de ida y vuelta de $20 \mathrm{~m}$. en ambos géneros son superiores con respecto a los obtenidos por Secchi et al. 2014).

Por último, indicar cómo la correlación existente entre la práctica de actividades extracurriculares con la mejora de fuerza y resistencia, va en la linea de los resultados obtenidos por Bahamonde, Carmona, Albomoz, Hernández-García \& Torres-Luque (2019), quienes obtienen beneficios a nivel cardiovascular y en salto de longitud tras aplicar un programa de 12 semanas de actividades físicas extraescolares en chilenos de 13-14 años.

\section{Conclusiones}

Los chicos tienen valores más saludables de $\mathrm{CF}$ en perimetro de cintura $\mathrm{y}$ mejores en fuerza de tren superior y resistencia. 
En todos los tramos de edad los hombres tienen mejor fuerza de tren superior e inferior y resistencia. En este género la fuerza se incrementa con la edad

Una mayor actividad física no implica una mejor CF. Sin embargo la práctica de deporte competitivo fuera del centro escolar sí mejora los valores de $\mathrm{CF}$ en la capacidad de resistencia y fuerza del tren inferior.

Según el estudio realizado, sólo la práctica competitiva extraescolar es la que está relacionada con la mejora de la resistencia cardiovascular y la fuerza, lo que implica la necesidad de revisar por parte del profesorado, qué y cómo se están trabajando los aspectos de $\mathrm{CF}$ en las clases de educación fisica.

Además, los altos índices de inactividad en las edades entre 13 y 17 años, requiere buscar estrategias para incrementar la práctica de AF dentro del centro escolar (no sólo en la clase de Educación Física sino también en los recreos, e incluso en otras asignaturas) y fuera de él, en todos los adolescentes y en especial en las chicas, que son, a su vez, las que peor resistencia cardiovascular tienen. Además se deben establecer mecanismos para reducir la inactividad y el sedentarismo, implicando más a los jóvenes, en actividades fisicas de ocio, tanto en deportes competitivos como en otras actividades que puedan resultarles más atractivas en función de las diferentes motivaciones (bailar, caminar, montar en bicicleta, actividades de fitness), pero que tengan una intensidad, duración y frecuencia suficiente como para que se puedan producir mejoras de componentes de $\mathrm{CF}$ que se relacionan con la salud.

\section{Referencias}

Ardoy, D.N., Fernández-Rodríguez, J.M., Ruiz, J.R., Chillón, P., EspañaRomero, V., Castillo, M.J. \& Ortega, F.B. (2011). Improving physical fitness in adolescents through a school-based intervention: the EDUFIT study. Revista Española de Cardiología, 64(6), 484-91. DOI: 10.1016/j.rec.2011.02.010

Arriscado, D., Muros, J., Zabala, M. \& Dalmau, J. (2014). Relación entre condición física y composición corporal en escolares de primaria del norte de España (Logroño). Deporte y ejercicio, 30(2), 385-394. http://dx.doi.org/10.3305/nh.2014.30.2.7217

Bahamonde, C. Carmona, C., Albomoz, J., Hernández-García, R. \& TorresLuque, G. (2019). Efecto de un programa de actividades deportivas extraescolares en jóvenes chilenos. Retos, 35, 261-266.

Balady, G.J. (2002). Survival of the fittest - more evidence. The New England Journal of Medicine, 346 (11), 852-854. DOI: 10.1056/NEJM200203143461111

Boreham C, Twisk J, Neville C, Savage M, Murray L. \& Gallagher A. (2002). Associations between physical fitness and activity patterns during 
adolescence and cardiovascular risk factors in young adulthood: the Northern Ireland Young Hearts Project. Int $J$ Sports Med., 23(1), S2226.

Bucco-dos Santos, L. \& Zubiaur-González, M. (2007). Desarrollo de las habilidades motoras fundamentales en función del sexo y del índice de masa corporal en escolares. Cuadernos de Psicología del Deporte, 13(2), 63-72.

Carriedo, A.; Cecchini, J.A. (2019). ¿Cómo aumentar la actividad física diaria dentro del horario escolar? Ejemplo de un proyecto de intervención interdisciplinar entre educación física y matemáticas. Journal of Sport and Health Research. 11(Supl 1):221-230

Catley, M.J. \& Tomkinson, G.R. (2013). Normative health-related fitness values for children: analysis of 85347 test results on 9-17-year-old Australians since 1985. Br $J$ Sports Med, 47(2), 98-108. DOI: 10.1136/bjsports-2011-090218

Cuenca-Garcia, M., Jiménez-Pavón, D., Artero, E.G., Castro-Piñero, J., Ortega, F.B., Ruiz, J.R. \& Castillo, M.J. (2011). Condición física relacionada con la salud y hábitos de alimentación en niños y adolescentes: propuesta de addendum al informe de salud escolar. Revista de Investigación en Educación, 9(2), 35-50.

Eisenmann, J.C. \& Malina, R.M. (2002). Secular trend in peak oxygen consumption among United Status youth in the 20th century. American Journal of Human Biology, 14, 699-706. https://doi.org/10.1002/ajhb.10084

España-Romero, V., Artero EG, Jiménez-Pavon, D., Cuenca-García, M., Ortega, F.B., Castro-Pinero, J., Sjostrom, M., Castillo-Garzón, M.J. \& Ruiz, J.R. (2010). Assessing health-related fitness tests in the school setting: reliability, feasibility and safety; the ALPHA Study. Int J Sports Med, 31(7), 490-497. DOI:10.3305/nh.2011.26.6.5611

Fundación para la Investigación Nutricional. (2018). Informe 2018: Actividad Física en niños y adolescentes en España. Fundación Para La Investigación Nutricional. Disponible en http://muchofit.com/wpcontent/uploads /2019/01/INFORME_2018.pdf

Nieto, L. García, E., \& Guillamón, A. (2020). Valores de Condición Física relacionada con la Salud en adolescentes de 14 a 17 años; relación con el estado de peso, Retos, 37, 215-221

Guthold, R., Stevens, G.A., Riley, L.M., \& Bull, F.C. (2019). Global trends in insufficient physical activity among adolescents: a pooled analysis of 298 population-based surveys with 1.6 million participants. The Lancet Child \& Adolescent Health, 4(1), 23-35. https:/ / doi.org/10.1016/S2352-4642(19)30323-2

Kim, J., Must, A., Fitzmaurice, G.M., Gillman, M.W., Chomitz, V., Kramer, E., McGowan, R. \& Peterson, K.E. (2005). Relationship of physical 
Condición física en jóvenes y su relación con la actividad física escolar y extraescolar

fitness to prevalence and incidence of overweight among
schoolchildren. Obesity Research, 13(7), 1246-1254. DOI: 10.1038/oby.2005.148

Laukkanen, J.A., Lakka, T.A., Rauramaa, R., Kuhanen, R., Venalainen, J.M., Salonen, R. \& Salonen, J.T. (2001). Cardiovascular fitness as a predictor of mortality in men. Arch Intern Med., 161(6), 825-831. DOI: 10.1001/archinte.161.6.825

Martínez-Gómez, D., Martínez-de-Haro, V., Pozo, T., Welk, G.J., Villagra, A., Calle, M.E., Marcos, A. \& Veiga, O.L. (2009). Fiabilidad y validez del cuestionario de actividad Física PAQ-A en adolescentes españoles. Rev Esp Salud Pública, 3(83), 427-439. http:/ / scielo.isciii.es/scielo.php?script=sci_arttext\&pid=S113557272009000300008

Martínez, J., de los Reyes-Corcuera, M., Borrell-Lizana, V. \& Pastor-Vicedo, J.C. (2018). Valoración de los niveles de condición física de escolares de 11-12 años, mediante a aplicación de la Batería ALPHAFITNES. SPORT TK. Revista Euroamericana de Ciencias del Deporte, 7(2), 3741. DOI: https://doi.org/10.6018/sportk.343211

Martínez-Vizcaíno, V. \& Sánchez-López, M. (2008). Relación entre actividad física y condición física en niños y adolescentes. Revista Española de Cardiología., 61(2),108-111. DOI: 10.1157/13116196

Merino, B. \& González, E. (2010). Actividad física y salud en la infancia y la adolescencia Guía para todas las personas que participan en su educación. Salud Pública. Promoción de la salud y epidemiología. Madrid: Ministerio de Educación y Ciencia. Ministerio de Sanidad y Consumo.

https://www.mscbs.gob.es/ciudadanos/proteccionSalud/adultos/act iFisica/docs/ActividadFisicaSaludEspanol.pdf

Muntaner-Mas, A., Vidal-Conti, J., Cantallops, J., Borràs, P.A., \& Palou, P. (2017). Obesity and physical activity patterns among Balearic Islands children and adolescents: a cross-sectional study. Journal of Human Sport and Exercise, 12(2), 333-348. doi:10.14198/jhse.2017.122.10

Moreno, L. A., Mesana, M. I., Fleta, J., Ruiz, J. R., Gonzalez-Gross, M., Sarria, A., Marcos, A. \& Bueno, M. (2005). Overweight, obesity and body fat composition in Spanish adolescents. The AVENA Study. Ann Nutr Metab, 49(2), 71-76. DOI: 10.1159/000084738

Organización Mundial de la Salud. (2010). Recomendaciones mundiales sobre actividad física para la salud. Ginebra: Organización Mundial de la Salud.

Ortega, F.B., Ruiz, J.R., Castillo, M.J., Moreno, L.A., Gonzalez-Gross, M., Warnberg, J. \& Gutierrez, A. (2005). Low level of physical fitness in Spanish adolescents. Relevance for future cardiovascular health (AVENA study). Revista Española de Cardiología, 58, 898-909. DOI: 
10.1016/S1885-5857(06)60372-1

Ortega, F.B., Tresaco, B., Ruiz, J.R., Moreno, L.A., Martin-Matillas, M., Mesa, J.L., Warnberg, J., Bueno, M., Tercedor, P., Gutiérrez, A., Castillo, M.J. \& AVENA Study Group (2007). Cardiorespiratory fitness and sedentary activities are associated with adiposity in adolescents. Obesity, 15, 1589-1599. DOI: 10.1038/oby.2007.188

Ortega, F.B., Ruiz, J.R., Hurtig-Wennlöf, A. \& Sjöström, M. (2008). Los adolescentes físicamente activos presentan más probabilidad de una capacidad cardiovascular saludable independientemente del grado de adiposidad. The European Youth Heart Study. Revista Española de Cardiologia, 61, 123-129. DOI: 10.1157/13116199

Ortega, F.B., Ruiz, J.R., Hurtig-Wennlof, A., Vicente-Rodriguez, G., Rizzo, N.S., Castillo, M.J. \& Sjöström, M. (2010). Cardiovascular fitness modifies the associations between physical activity and abdominal adiposity in children and adolescents: the European Youth Heart Study. $B r \quad J \quad$ Sports Med., 44, 256-262. http:/ /dx.doi.org/10.15366/rimcafd2016.62.007

Ortega, F.B., Ruiz, J.R. \& Castillo, M.J. (2013). Actividad fisica, condición física y sobrepeso en niños y adolescentes: evidencia procedente de estudios epidemiológicos. Endocrinología y Nutrición, 60(8), 458-469. https://doi.org/10.1016/j.endonu.2012.10.006

Physical Activity Guidelines Advisory Committee. (2018). Physical Activity Guidelines Advisory Committee Scientific Report. Washington, DC: US Department of Health and Human Services. https://health.gov/paguidelines/secondedition/report/pdf/PAG_Advisory_Committee_Report.pdf

Ruiz, J.R., España-Romero, V., Castro-Piñero, J., Artero, E.G., Ortega, F.B., Cuenca-García, M., Jiménez-Pavón, D., Chillón, P., GirelaRejón, M.J., Mora, J., Gutiérrez, A., Suni, J., Sjöstrom, M. \& Castillo, M.J. (2011). Batería ALPHA-Fitness: test de campo para la evaluación de la condición física relacionada con la salud en niños y adolescentes. Nutrición Hospitalaria, 26 (6), 1210-1214. DOI: $10.3305 / \mathrm{nh} .2011 .26 .6 .5611$

Ruiz, J.R., Ortega, F.B., Gutierrez, A., Meusel, D., Sjöström, M. \& Castillo, M.J. (2006). Health-related fitness assessment in childhood and adolescence; A European approach based on the AVENA, EYHS and HELENA studies. Journal of Public Health, 14, 269-277. DOI: $10.1007 / \mathrm{s} 10389-006-0059-z$

Ruiz, E., Ávila, J.M., Castillo, A., Valero, T., Del Pozo, S. \& Rodríguez, P. (2015). The ANIBES study on Energy Balance in Spain: design, protocol and methodology. Nutrients, 7, 970-998. DOI: $10.3390 / \mathrm{nu} 7020970$.

Ruiz, J. R., Castro-Pinero, J., Artero, E. G., Ortega, F. B., Sjostrom, M., 
Suni, J. \& Castillo, M. J. (2009). Predictive validity of health-related fitness in youth: a systematic review. British Journal of Sports Medicine, 43(12), 909-923. DOI: 10.1136/bjsm.2008.056499

Rush, E., McLennan, S., Obolonkin, V., Vandal, A.C., Hamlin, M., Simmons, D. \& Graham, D. (2014). Project Energize: whole-region primary school nutrition and physical activity programme; evaluation of body size and fitness 5 years after the randomised controlled trial. British Journal of Nutrition, 111(2), 363-371. DOI: $10.1017 /$ S0007114513002316

Sallis, J.F. (2000). Age-related decline in physical activity: a synthesis of human and animal studies. Medicine \& Science in Sports \& Exercise, 32,1598-1600. DOI: 10.1097/00005768-200009000-00012

Secchi, J.D., García, G.C., España-Romero, V. \& Castro-Piñero, J. (2014). Condición fisica y riesgo cardiovascular futuro en niños y adolescentes argentinos: una introducción de la batería ALPHA. Arch Argent Pediatr., 112(2), 132-140. https://www.sap.org.ar/docs/publicaciones/archivosarg/2014/v112n 2a05.pdf

Seguin, R. \& Nelson, M.E. (2003). The benefits of strength training for older adults. American Journal of Preventive Medicine., 25, S141-149. DOI:10.1016/s0749-3797(03)00177-6

Serra-Majem, L., Ribas-Barba, L., Aranceta, J., Pérez-Rodrigo, C., SaavedraSantana, P. \& Pena-Quintana, L. (2003). [Childhood and adolescent obesity in Spain. Results of the enKid study (1998-2000)]. Med Clin (Barc), 121(19), 725-732. DOI: 10.1016/s0025-7753(03)74077-9

Sylejmani, B., Myrtaj, N., Maliqi, A., Gontarev, S., Georgiev, G., \& Kalac, R. (2019). Physical fitness in children and adolescents in rural and urban areas. Journal of Human Sport and Exercise, 14(4), 866- 875. doi:https://doi.org/10.14198/jhse.2019.144.15

Tomkinson, G.R., Léger, L.A., Olds, T.S. \& Cazorla, G. (2003). Secular trends in the performance of children and adolescents (1980-2000). An analysis of 55 studies of the $20 \mathrm{~m}$ shuttle run test in 11 countries. Sports Medicine, 33, 285-300. DOI: 10.2165/00007256-20033304000003 
114 ISSN0213-8646| E-ISSN2530-3791 • Revista Interuniversitaria de Formación delProfesorado, 95 (34.1) (2020), 99-114 